\title{
A novel and simple method for estimating the fractional Raman contribution
}

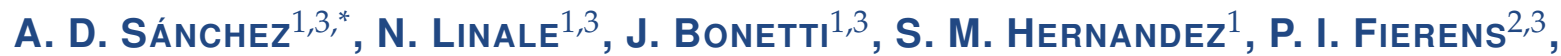 \\ G. BRAMBILLA ${ }^{4}$, AND D. F. GRosz ${ }^{1,3}$ \\ ${ }^{1}$ Grupo de Comunicaciones Ópticas, Instituto Balseiro,Bariloche, Río Negro 8400, Argentina \\ ${ }^{2}$ Grupo de Optoelectrónica, Instituto Tecnológico de Buenos Aires, CABA 1106, Argentina \\ ${ }^{3}$ Consejo Nacional de Investigaciones Científicas y Técnicas (CONICET), Argentina \\ ${ }^{4}$ Optoelectronics Research Centre, University of Southampton SO17 1BJ, England \\ *Corresponding author: alfredo.sanchez@ib.edu.ar
}

Compiled December 12, 2018

We propose a novel and simple method for estimating the fractional Raman contribution, $f_{R}$, based on an analysis of a full model of modulation instability (MI) in waveguides. An analytical expression relating $f_{R}$ to the MI peak gain beyond the cutoff power is explicitly derived, allowing for an accurate estimation of $f_{R}$ from a single measurement of the Raman gain spectrum. () 2018 Optical Society of America

OCIS codes: (190.5650) Raman effect; (190.4380) Nonlinear optics, fourwave mixing

\section{http://dx.doi.org/10.1364/ao.XX.XXXXXX}

Stimulated Raman scattering (SRS) is one of the most prominent phenomena in nonlinear optics and may occur in any molecular medium. SRS can be regarded as a four-photon process leading to the transfer of energy from a pump wave to lower frequency (Stokes) and higher frequency (anti-Stokes) waves through the mediation of an optical phonon provided by the transmission medium [1]. In particular, optical fibers have enabled the fabrication of broadband amplifiers [2] and tunable lasers [3] based on SRS. In media exhibiting third-order susceptibility $\chi^{(3)}$, the Raman response function is usually modeled with two dominant time scales (see, e.g., [1]). The shorter time scale is due to nonresonant virtual electronic transitions and, for most applications, can be modeled as an instantaneous Dirac's delta. The longer time scale, on the other hand, is due to the nuclear contribution of the interaction. All in all, the total Raman response $R(t)$ can be written as

$$
R(t)=\left(1-f_{R}\right) \delta(T)+f_{R} h_{R}(T),
$$

where $f_{R}$ weights the relative contributions of the instantaneous and delayed Raman responses. $R(t)$ and $h_{R}(t)$ are both normalized so that $\int_{\mathbb{R}} R(t) d t=\int_{\mathbb{R}} h_{R}(t) d t=1$. The function $h_{R}(t)$ can be estimated from experiments by relating it to the Raman gain spectrum $g_{R}$ of the waveguide [4-6]:

$$
g_{R}(\Omega)=f_{R} \frac{2 \omega_{0}}{c} n_{2}\left(\omega_{0}\right) \operatorname{Im}\left\{\tilde{h}_{R}(\Omega)\right\}
$$

where $\omega_{0}$ is the pump frequency, $\Omega$ denotes frequency deviations from $\omega_{0}, c$ is the speed of light, $n_{2}$ is the nonlinear refractive index, and $\tilde{h}_{R}(\Omega)$ is the Fourier transform of $h_{R}(t)$.

Given an estimation of $\tilde{h}_{R}(\Omega)$, the fraction of the delayed Raman response $f_{R}$ can be computed provided an independent measurement of the nonlinear refractive index $n_{2}$. This is the chosen procedure, for instance, by $\mathrm{Hu}$ and colleagues $[5,6]$ in the case of $\mathrm{As}_{2} \mathrm{Se}_{3}$ chalcogenide fibers. It is interesting to note that Refs. [5, 6] use measurements in Slusher et al. [7]. The authors of Ref. [7] remark the difficulties of obtaining precise values, as their "experimental values for both $n_{2}$ and $g_{R}$ have at least $30 \%$ errors due primarily to the uncertainties in evaluating the effective power and intensity in the fiber." As such, experimental uncertainties hinder a precise estimation of $f_{R}$.

Other ways of estimating the fractional contribution $f_{R}$ have been presented in the literature. Hellwarth et al. [8] resort to measurements of intensity-induced polarization changes and the Raman differential scattering cross section to determine $h_{R}(t)$ and $f_{R}$ for several glasses, including fused quartz. In the seminal work by Stolen and colleagues [4,9] for fused silica, a relation between the Raman gain and the differential scattering cross section is used. Since independent measurements of both quantities are possible, $f_{R}$ can be calculated. The estimated value of $f_{R}=0.18$ is the one commonly adopted for silica-based fibers [4].

In numerical simulations, $h_{R}(t)$ is usually replaced by a simple mathematical expression and not derived from experimental measurements. The most common approach is to fit $g_{R}(\Omega)$ (and hence $\tilde{h}_{R}(\Omega)$ ) with a Lorentzian profile (damped-oscillator approximation in the time domain) $[1,10]$. Furthermore, $f_{R}$ is sometimes estimated from such a fit and Eq. (2) (see, e.g., [11]). However, a single Lorentzian linewidth does not suffice to properly describe the spectral characteristics of the Raman gain, and more complex models are used to compute the gain at various frequencies. One such model for silica fibers was put forth by Lin and Agrawal [12]. In particular, they describe the so called boson peak of the Raman response in silica fibers. However, the resulting model overestimates the Raman gain in the spectral region beyond $15 \mathrm{THz}$, causing an underestimation of the electronic contribution to the nonlinear refractive index. For 
this reason, Lin and Agrawal set $f_{R}=0.245$, higher than the experimentally measured value $f_{R}=0.18$, to compensate.

There are more complex approximate mathematical expressions for $h_{R}(t)$. A usual approach is that proposed by Hollenbeck and Cantrell [13] which is based on the superposition of Gaussian-broadened Lorentzian linewidths. The authors suggest that this type of approximation allows for a better fit of both the gain spectrum and the response $h_{R}(t)$ than the usual singleLorentzian-profile approach. They show how their approach works for silica fibers, although they do not estimate $f_{R}$. Other authors have used the work in Ref. [13] to fit the Raman gain and response for different fiber types, including the estimation of the fractional electronic contribution. For instance, Yan et al. [14] estimated values for ZBLAN fibers and Kohoutek et al. [15] used them for $\mathrm{As}_{2} \mathrm{~S}_{3}$ and $\mathrm{Ge}_{17} \mathrm{Ga}_{4} \mathrm{Sb}_{10} \mathrm{~S}_{69}$ glasses. There also are other approximate analytical expressions for $h_{R}(t)$. Agger et al. [16], e.g., fit two Gaussian profiles to the gain $g_{R}(\Omega)$ and estimate $f_{R}$ from Eq. (2) for ZBLAN fibers.

The nonlinear refraction index $n_{2}$ can be measured through the Z-scan technique $[17,18]$. In particular, time-resolved Zscans allow the separation of the electronic and nuclear contributions to $R(t)$ in the time domain. Indeed, $R(t)$ can be directly estimated from measurements performed with this technique. Smolorz et al. [19] use time-resolved Z scans to estimate $h_{R}(t)$ and $f_{R}$ in several chalcogenide and heavy-metal glasses. It must be noted that the authors report errors $>25 \%$ for $f_{R}$.

It is usual in the literature to resort to measurements and estimations in previous works, even though the particular fiber might not be made of exactly the same material. The reason for this is that an accurate estimation of, say, $f_{R}$ requires a considerable experimental effort in the lines of the aforementioned studies. In some cases, researchers adopt published values with slight changes to better fit experimental observations. For example, Duhant et al. [20] work with a $\mathrm{As}_{38} \mathrm{Se}_{62}$ suspended-core microstructured fiber. Since there are no previous studies with this material, to describe the Raman response they modify values in Ref. [21] for $\mathrm{As}_{2} \mathrm{Se}_{3}$ in order to fit their observations. As Duhant and colleagues observe, "the exact Raman response of AsSe glass is not yet fixed accurately in the available literature".

In short, the experimental estimation of the fractional electronic contribution to the Raman response is usually difficult and is accompanied with errors of the order of $20-30 \%$. Moreover, accurate assessments of $f_{R}$ for new materials are lacking.

In this work, we propose a novel and simple technique based on a relatively unexplored facet of modulation instability (MI). In the absence of delayed Raman scattering $\left(f_{R}=0\right)$ it can be shown that, if the effect of self-steepening is considered, the MI gain vanishes when the pump power exceeds a certain limit $[22,23]$. However, if $f_{R}>0$, there is still gain beyond the cutoff power. Furthermore, we have shown [24, 25] that the MI gain takes a Raman-like shape with a power-tunable central frequency. As we shall demonstrate, the way in which this central frequency varies with the pump power depends on the value of $f_{R}$. It is this dependence that can be exploited to obtain an accurate estimation of the fractional Raman contribution to the response. Using the fact that $\tilde{h}_{R}(\Omega)$ is analytic in the upper halfplane, the Kramers-Kronig relations enable the calculation of the real part of $\tilde{h}_{R}(\Omega)$ and, through the Fourier anti-transform, the computation of $h_{R}(t)$. An example of this calculation is shown in Fig. 1 where the Raman response is obtained from our back-scattering measurements of a sample of $\mathrm{As}_{2} \mathrm{~S}_{3}[26,27]$ chalcogenide.

In order to explain our approach to the estimation of $f_{R}$,
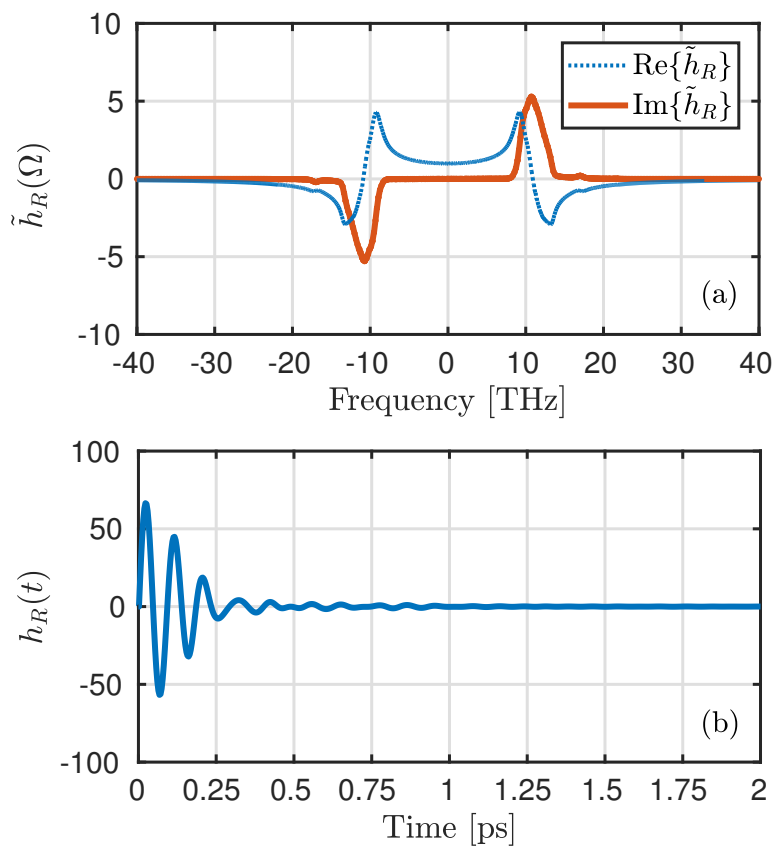

Fig. 1. Measured Raman spectrum of a chalcogenide $\mathrm{As}_{2} \mathrm{~S}_{3}$ optical fiber. (a) Real and imaginary parts of $\tilde{h}_{R}(\Omega)$. (b) Time response $h_{R}(t)$.

we start by the generalized nonlinear Schrödinger equation (GNLSE) [10], used to describe propagation of short pulses in a lossless monomode nonlinear waveguide

$\frac{\partial A(z, T)}{\partial z}-i \hat{\beta} A(z, T)=i \hat{\gamma} A(z, T) \int_{-\infty}^{\infty} R\left(T^{\prime}\right)\left|A\left(z, T-T^{\prime}\right)\right|^{2} d T^{\prime}$,

where $A(z, T)$ is the slowly-varying envelope, $z$ is the spatial coordinate, and $T$ is the time coordinate in a co-moving frame at the group velocity. The operator $\hat{\beta}$ models the linear dispersion and $\hat{\gamma}$ is an operator related to the third-order susceptibility, and the integral on the right hand side includes the influence of Raman scattering. Operators $\hat{\beta}$ and $\hat{\gamma}$ are defined as

$$
\hat{\beta}=\sum_{m \geq 2} \frac{i^{m}}{m !} \beta_{m} \frac{\partial^{m}}{\partial T^{m}}, \quad \hat{\gamma}=\sum_{n \geq 0} \frac{i^{n}}{n !} \gamma_{n} \frac{\partial^{n}}{\partial T^{n}} .
$$

Coefficients $\beta_{m}$ correspond to the Taylor expansion of the propagation constant $\beta(\omega)$ around a central frequency $\omega_{0}$. Similarly, $\gamma_{n}$ are the coefficients of the Taylor expansion of the nonlinear parameter. Usually is sufficient to consider the expansion up to the first term. Under this setting, it can be shown that the total number of photons is conserved if $\gamma_{1}=\gamma_{0} / \omega_{0}$ [1], which is the usual approximation.

It is well-known that, from a first-order linear perturbation analysis of the GNLSE, continuous-wave (CW) solutions become unstable upon propagation in the nonlinear medium. This phenomenon, known as modulation instability [28-35], is a parametric process where two photons from a CW pump are transferred to both low- and high-frequency bands, one photon each. As a result, the MI gain is observed at both frequency sides of the pump. A complete analysis including the rich interplay between MI and Raman scattering can be found in Refs. [25, 36, 37]. For 


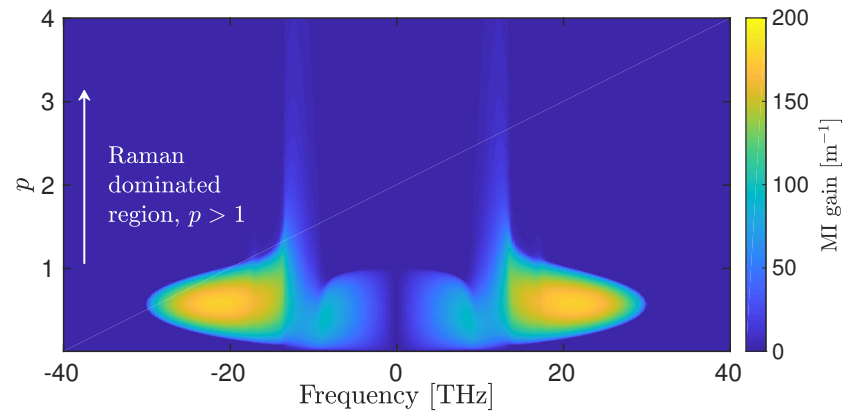

Fig. 2. MI gain profile vs. $p$ and frequency. The pump frequency was chosen $\omega_{0} / 2 \pi=29.98 \mathrm{THz}, \beta_{2}=-10 \mathrm{ps}^{2} / \mathrm{km}, \gamma_{0}=100$ $\mathrm{W}^{-1} \mathrm{~km}^{-1}$, and $f_{R}=0.1$.

the sake of simplicity, in what follows we only consider the case where $m=2$ and $n=1$ in Eq. (4). In this scenario, it can be shown that the the MI gain in the anomalous dispersion regime is given by $[24,38]$

$$
\begin{aligned}
g_{\mathrm{MI}}(\Omega) & =2 \max \left\{-\operatorname{Im}\left\{K_{1}(\Omega)\right\},-\operatorname{Im}\left\{K_{2}(\Omega)\right\}, 0\right\}, \\
K_{1,2}(\Omega, p) & =\frac{p\left|\beta_{2}\right|}{\tau} \Omega(1+\tilde{R}) \pm\left|\beta_{2} \Omega\right| \sqrt{\frac{\Omega^{2}}{4}-\frac{p \tilde{R}}{\tau^{2}}+\frac{p^{2} \tilde{R}^{2}}{\tau^{2}}},
\end{aligned}
$$

where $\tau=\gamma_{1} / \gamma_{0}, \tilde{R}(\Omega)$ is the Fourier transform of $R(t)$ and $p=P_{0} / P_{\mathrm{C}}$ is the normalized pump power, with $P_{\mathrm{C}}$ defined as the power cutoff

$$
P_{\mathrm{c}}=\frac{\left|\beta_{2}\right| \gamma_{0}}{\gamma_{1}^{2}}
$$

As aforementioned, in the absence of Raman scattering $\left(f_{R}=0\right)$ it is easy to verify that the MI gain vanishes for $p>1$. However, in the presence of Raman scattering $\left(f_{R} \neq 0\right)$ there is MI gain for $p>1$, and its profile changes drastically. Figure 2 shows the MI gain profile from Eq. (5), using the Raman spectrum in Fig. 1 and assuming $f_{R}=0.1$, where the region for $p>1$ is clearly dominated by Raman.

The position of the MI peak gain, $\Omega_{\mathrm{MI}}$, tends to remain stable as the pump power increases. Since Eq. (6) does not lend to a simple algebraic manipulation, it is more convenient to work with the asymptotic limit of $g_{\mathrm{MI}}$, expressed as

$$
\lim _{p \rightarrow \infty} g_{\mathrm{MI}}(\Omega, p) \equiv g_{\mathrm{MI}}^{\infty} \propto|\Omega|\left|\operatorname{Im}\left\{\tilde{R}^{-1}\right\}\right| .
$$

Beyond the cutoff power $(p \gg 1)$, the shape of $g_{\mathrm{MI}}^{\infty}$ depends only on the Raman characteristics of the transmission medium. From a significant number of numerical simulations, we verified that this is satisfied for $p \geq 5$. Figure 3 shows the gain profile $g_{\mathrm{MI}}$ for $p=10$ (a), and the dependence of $\Omega_{\mathrm{MI}}$ with $p$ for several values of $f_{R}(\mathrm{~b})$.

The location of the MI peak can be found by setting $\partial_{\Omega} g_{M I}^{\infty}=$ 0 . After some algebraic manipulations, it can be shown that $f_{R}$ is the solution in $(0,1)$ of the quadratic equation

$$
a f_{R}^{2}+b f_{R}+c=0
$$

with

$$
\begin{aligned}
a & =\partial_{\Omega}|\tilde{h}|^{2}-2 \partial_{\Omega} \tilde{h}^{R}-\frac{\tilde{h}^{I}+\Omega \partial_{\Omega} \tilde{h}^{I}}{\Omega \tilde{h}^{I}}\left(1+|\tilde{h}|^{2}-2 \tilde{h}^{R}\right) \mathbf{( 1 0 )} \\
b & =2 \partial_{\Omega} \tilde{h}^{R}-\frac{\tilde{h}^{I}+\Omega \partial_{\Omega} \tilde{h}^{I}}{\Omega \tilde{h}^{I}}\left(2 \tilde{h}^{R}-2\right) \\
c & =-\frac{\tilde{h}^{I}+\Omega \partial_{\Omega} \tilde{h}^{I}}{\Omega \tilde{h}^{I}}
\end{aligned}
$$
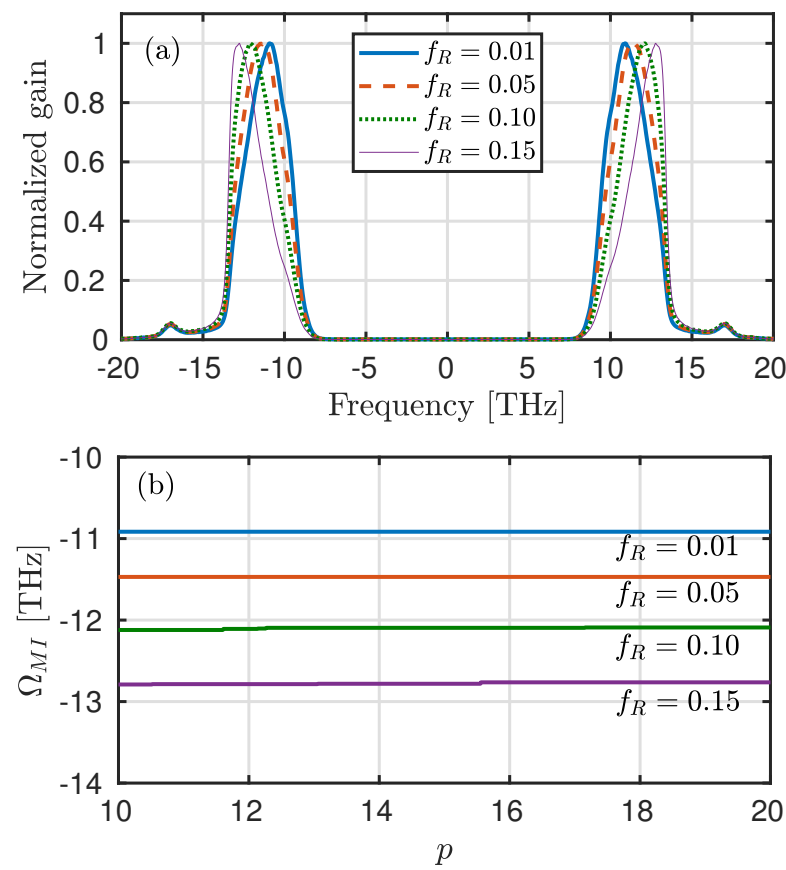

Fig. 3. Normalized $g_{\text {MI }}$ profile with $p=10$ (a) and $\Omega_{\text {MI }}$ vs. $p$ on the Stokes side (b) for several values of $f_{R}$.

where $\tilde{h}^{R}$ and $\tilde{h}^{I}$ stand for the real and imaginary parts of $\tilde{h}=$ $\tilde{h}_{R}(\Omega)$, respectively, and all quantities are evaluated at $\Omega=$ $\Omega_{\mathrm{MI}}$. Numerical results are obtained by solving the GNLSE with a fourth-order Runge-Kutta Interaction Picture algorithm [39]. Figure 4(a) shows the evolution of a pump with an additive white Gaussian noise. For these simulations we assumed an $f_{R}$ around 0.1 , consistent with that from Ref. [11]. The peak position $\Omega_{\mathrm{MI}}$ can be obtained from these spectra, and then $f_{R}\left(\Omega_{\mathrm{MI}}\right)$ can be estimated using Eq. (9). Note that in an actual experiment, one would perform a measurement of the spectrum by pumping the waveguide (ensuring that the condition $p \geq 5$ is met) and obtain the MI peak. Figure 4(b) shows the dependence of $f_{R}$ with $\Omega_{\text {MI }}$ from Eq. (9). Table 1 shows a comparison between simulations (for different $f_{R}$ ) and the corresponding $f_{R}$ obtained with the proposed method. As the table indicates, relative errors are less than $10 \%$. Note that in experimental conditions an additional error may be incurred from the finite resolution of the measuring instrument.

Table 1. Estimation of $f_{R}$.

\begin{tabular}{cccc}
\hline $\begin{array}{c}\Omega_{\mathrm{MI}} \\
{[\mathrm{THz}]}\end{array}$ & $f_{R}^{\text {Simulation }}$ & $f_{R}^{\text {Estimated }}$ & Relative error \\
\hline 10.95 & 0.010 & 0.011 & $9 \%$ \\
11.45 & 0.050 & 0.048 & $4 \%$ \\
12.11 & 0.100 & 0.102 & $2 \%$ \\
12.90 & 0.150 & 0.159 & $6 \%$ \\
\hline
\end{tabular}

In summary, this work puts in evidence the way the Raman 

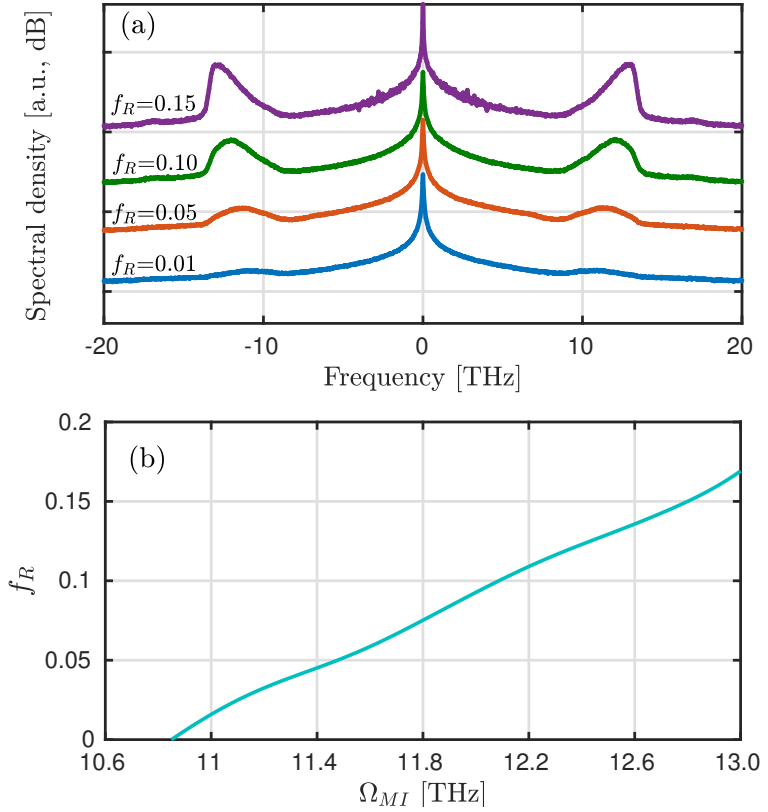

Fig. 4. (a) Simulation results of a CW pump with additive white Gaussian noise, for an average over 200 noise realizations, and for several values of $f_{R}$. The CW pump frequency and the optical fiber parameters are the same as those in Fig. 3. The propagation length is $L=2.5 \mathrm{~m}$. A normalized pump power $p=10$ and an initial pump-to-noise ratio of $30 \mathrm{~dB}$ are assumed. (b) $f_{R}$ vs. $\Omega_{\mathrm{MI}}$ from Eq. (9).

fractional contribution $f_{R}$ affects the asymptotic position of the MI peak gain $\Omega_{\mathrm{MI}}$ in a full model of modulation instability in waveguides. An analytical expression relating $f_{R}$ to $\Omega_{\mathrm{MI}}$ was derived, for the first time to the best of our knowledge, allowing for an accurate and novel way to estimate $f_{R}$ from a single measurement of the Raman gain spectrum.

Acknowledgment: We gratefully acknowledge P. Soubelet for the measurement of the Raman spectrum of the chalcogenide fiber, and NVIDIA Corporation for the kind donation of a Tesla K40 GPU card.

\section{REFERENCES}

1. K. Blow and D. Wood, IEEE J. Quantum Electron. 25, 2665 (1989).

2. M. N. Islam, IEEE J. selected topics Quantum Electron. 8, 548 (2002).

3. C. Lin, R. H. Stolen, W. G. French, and T. G. Malone, Opt. Lett. 1, 96 (1977).

4. R. H. Stolen, W. J. Tomlinson, H. A. Haus, and J. P. Gordon, J. Opt. Soc. Am. B 6, 1159 (1989).

5. J. Hu, C. R. Menyuk, L. B. Shaw, J. S. Sanghera, and I. D. Aggarwal, "Raman response function and supercontinuum generation in chalcogenide fiber," in Conference on Lasers and Electro-Optics/Quantum Electronics and Laser Science Conference and Photonic Applications Systems Technologies, (Optical Society of America, 2008), p. CMDD2.

6. J. Hu, C. R. Menyuk, L. B. Shaw, J. S. Sanghera, and I. D. Aggarwal, Opt. Express 18, 6722 (2010).

7. R. E. Slusher, G. Lenz, J. Hodelin, J. Sanghera, L. B. Shaw, and I. D. Aggarwal, J. Opt. Soc. Am. B 21, 1146 (2004).

8. R. Hellwarth, J. Cherlow, and T.-T. Yang, Phys. Rev. B 11, 964 (1975).

9. R. H. Stolen and E. P. Ippen, Appl. Phys. Lett. 22, 276 (1973).

10. G. Agrawal, Nonlinear Fiber Optics, Optics and Photonics (Academic Press, 2012), 5th ed.
11. C. Xiong, E. Magi, F. Luan, A. Tuniz, S. Dekker, J. S. Sanghera, L. B. Shaw, I. D. Aggarwal, and B. J. Eggleton, Appl. Opt. 48, 5467 (2009).

12. Q. Lin and G. P. Agrawal, Opt. Lett. 31, 3086 (2006).

13. D. Hollenbeck and C. D. Cantrell, J. Opt. Soc. Am. B 19, 2886 (2002).

14. X. Yan, C. Kito, S. Miyoshi, M. Liao, T. Suzuki, and Y. Ohishi, J. Opt. Soc. Am. B 29, 238 (2012).

15. T. Kohoutek, X. Yan, T. W. Shiosaka, S. N. Yannopoulos, A. Chrissanthopoulos, T. Suzuki, and Y. Ohishi, J. Opt. Soc. Am. B 28, 2284 (2011).

16. C. Agger, C. Petersen, S. Dupont, H. Steffensen, J. K. Lyngsø, C. L. Thomsen, J. Thøgersen, S. R. Keiding, and O. Bang, JOSA B 29, 635 (2012).

17. M. Sheik-bahae, A. A. Said, and E. W. V. Stryland, Opt. Lett. 14, 955 (1989).

18. J. Wang, M. Sheik-Bahae, A. A. Said, D. J. Hagan, and E. W. V. Stryland, J. Opt. Soc. Am. B 11, 1009 (1994).

19. S. Smolorz, I. Kang, F. Wise, B. Aitken, and N. Borrelli, J. NonCrystalline Solids 256-257, 310 (1999).

20. M. Duhant, W. Renard, G. Canat, T. N. Nguyen, F. Smektala, J. Troles, Q. Coulombier, P. Toupin, L. Brilland, P. Bourdon, and G. Renversez, Opt. Lett. 36, 2859 (2011).

21. B. Ung and M. Skorobogatiy, Opt. Express 18, 8647 (2010).

22. P. K. Shukla and J. J. Rasmussen, Opt. Lett. 11, 171 (1986).

23. C. D. Angelis, G. Nalesso, and M. Santagiustina, J. Opt. Soc. Am. B 13, 848 (1996).

24. A. D. Sánchez, S. M. Hernandez, J. Bonetti, P. I. Fierens, and D. F. Grosz, J. Opt. Soc. Am. B 35, 95 (2018).

25. A. D. Sánchez, P. I. Fierens, S. M. Hernandez, J. Bonetti, G. Brambilla, and D. F. Grosz, J. Opt. Soc. Am. B 35, 2828 (2018).

26. P. Wang, G. Brambilla, M. Ding, X. Zhang, Y. Semenova, Q. Wu, and G. Farrell, "An sms fiber structure based on chalcogenide multimode fiber," in Nonlinear Optics and Applications VI, , vol. 8434 (International Society for Optics and Photonics, 2012), p. 84340N.

27. P. Wang, G. S. Murugan, G. Brambilla, M. Ding, Y. Semenova, Q. Wu, and G. Farrell, IEEE Photon. Technol. Lett 24, 1103 (2012).

28. A. Hasegawa and W. Brinkman, IEEE J. Quantum Electron. 16, 694 (1980).

29. K. Tai, A. Hasegawa, and A. Tomita, Phys. Rev. Lett. 56, 135 (1986).

30. A. Demircan and U. Bandelow, Opt. Commun. 244, 181 (2005).

31. J. M. Dudley, G. Genty, F. Dias, B. Kibler, and N. Akhmediev, Opt. Express 17, 21497 (2009).

32. D. Solli, C. Ropers, P. Koonath, and B. Jalali, Nature 450, 1054 (2007).

33. K. Hammani, C. Finot, B. Kibler, and G. Millot, Photonics Journal, IEEE 1, 205 (2009).

34. N. Akhmediev, J. M. Soto-Crespo, and A. Ankiewicz, Phys. Rev. A 80, 043818 (2009).

35. S. T. Sørensen, C. Larsen, U. Møller, P. M. Moselund, C. L. Thomsen, and O. Bang, J. Opt. Soc. Am. B 29, 2875 (2012).

36. P. Béjot, B. Kibler, E. Hertz, B. Lavorel, and O. Faucher, Phys. Rev. A 83, 013830 (2011).

37. J. Bonetti, S. M. Hernandez, P. I. Fierens, and D. F. Grosz, Phys. Rev. A 94, 033826 (2016).

38. S. M. Hernandez, P. I. Fierens, J. Bonetti, A. D. Sánchez, and D. F. Grosz, IEEE Photonics J. 9, 1 (2017).

39. A. Rieznik, A. Heidt, P. Konig, V. Bettachini, and D. Grosz, IEEE Photonics J. 4, 552 (2012). 


\section{FULL REFERENCES}

1. K. Blow and D. Wood, "Theoretical description of transient stimulated Raman scattering in optical fibers," IEEE J. Quantum Electron. 25, 2665-2673 (1989).

2. M. N. Islam, "Raman amplifiers for telecommunications," IEEE J. selected topics Quantum Electron. 8, 548-559 (2002).

3. C. Lin, R. H. Stolen, W. G. French, and T. G. Malone, "A cw tunable near-infrared (1.085-1.175- $\mu \mathrm{m})$ raman oscillator," Opt. Lett. 1, 96-97 (1977).

4. R. H. Stolen, W. J. Tomlinson, H. A. Haus, and J. P. Gordon, "Raman response function of silica-core fibers," J. Opt. Soc. Am. B 6, 11591166 (1989).

5. J. Hu, C. R. Menyuk, L. B. Shaw, J. S. Sanghera, and I. D. Aggarwal, "Raman response function and supercontinuum generation in chalcogenide fiber," in Conference on Lasers and Electro-Optics/Quantum Electronics and Laser Science Conference and Photonic Applications Systems Technologies, (Optical Society of America, 2008), p. CMDD2.

6. J. Hu, C. R. Menyuk, L. B. Shaw, J. S. Sanghera, and I. D. Aggarwal, "Maximizing the bandwidth of supercontinuum generation in as_2se_3 chalcogenide fibers," Opt. Express 18, 6722 (2010).

7. R. E. Slusher, G. Lenz, J. Hodelin, J. Sanghera, L. B. Shaw, and I. D. Aggarwal, "Large raman gain and nonlinear phase shifts in high-purity as2se3 chalcogenide fibers," J. Opt. Soc. Am. B 21, 1146-1155 (2004).

8. R. Hellwarth, J. Cherlow, and T.-T. Yang, "Origin and frequency dependence of nonlinear optical susceptibilities of glasses," Phys. Rev. B 11, 964 (1975).

9. R. H. Stolen and E. P. Ippen, "Raman gain in glass optical waveguides," Appl. Phys. Lett. 22, 276-278 (1973).

10. G. Agrawal, Nonlinear Fiber Optics, Optics and Photonics (Academic Press, 2012), 5th ed.

11. C. Xiong, E. Magi, F. Luan, A. Tuniz, S. Dekker, J. S. Sanghera, L. B. Shaw, I. D. Aggarwal, and B. J. Eggleton, "Characterization of picosecond pulse nonlinear propagation in chalcogenide $\mathrm{As}_{2} \mathrm{~S}_{3}$ fiber," Appl. Opt. 48, 5467-5474 (2009).

12. Q. Lin and G. P. Agrawal, "Raman response function for silica fibers," Opt. Lett. 31, 3086-3088 (2006).

13. D. Hollenbeck and C. D. Cantrell, "Multiple-vibrational-mode model for fiber-optic Raman gain spectrum and response function," J. Opt. Soc. Am. B 19, 2886-2892 (2002).

14. X. Yan, C. Kito, S. Miyoshi, M. Liao, T. Suzuki, and Y. Ohishi, "Raman transient response and enhanced soliton self-frequency shift in zblan fiber," J. Opt. Soc. Am. B 29, 238-243 (2012).

15. T. Kohoutek, X. Yan, T. W. Shiosaka, S. N. Yannopoulos, A. Chrissanthopoulos, T. Suzuki, and Y. Ohishi, "Enhanced raman gain of ge-ga-sb-s chalcogenide glass for highly nonlinear microstructured optical fibers," J. Opt. Soc. Am. B 28, 2284 (2011)

16. C. Agger, C. Petersen, S. Dupont, H. Steffensen, J. K. Lyngsø, C. L. Thomsen, J. Thøgersen, S. R. Keiding, and O. Bang, "Supercontinuum generation in zblan fibers-detailed comparison between measurement and simulation," JOSA B 29, 635-645 (2012).

17. M. Sheik-bahae, A. A. Said, and E. W. V. Stryland, "High-sensitivity, single-beam n_2 measurements," Opt. Lett. 14, 955 (1989).

18. J. Wang, M. Sheik-Bahae, A. A. Said, D. J. Hagan, and E. W. V. Stryland, "Time-resolved z-scan measurements of optical nonlinearities," J. Opt. Soc. Am. B 11, 1009 (1994).

19. S. Smolorz, I. Kang, F. Wise, B. Aitken, and N. Borrelli, "Studies of optical non-linearities of chalcogenide and heavy-metal oxide glasses," J. Non-Crystalline Solids 256-257, $310-317$ (1999).

20. M. Duhant, W. Renard, G. Canat, T. N. Nguyen, F. Smektala, J. Troles, Q. Coulombier, P. Toupin, L. Brilland, P. Bourdon, and G. Renversez, "Fourth-order cascaded raman shift in AsSe chalcogenide suspendedcore fiber pumped at $2 \mu \mathrm{m}$," Opt. Lett. 36, 2859 (2011).

21. B. Ung and M. Skorobogatiy, "Chalcogenide microporous fibers for linear and nonlinear applications in the mid-infrared," Opt. Express 18, 8647-8659 (2010).

22. P. K. Shukla and J. J. Rasmussen, "Modulational instability of short pulses in long optical fibers," Opt. Lett. 11, 171-173 (1986).

23. C. D. Angelis, G. Nalesso, and M. Santagiustina, "Role of nonlinear dispersion in the dynamics of induced modulational instability in kerr media," J. Opt. Soc. Am. B 13, 848-855 (1996).

24. A. D. Sánchez, S. M. Hernandez, J. Bonetti, P. I. Fierens, and D. F. Grosz, "Tunable raman gain in mid-ir waveguides," J. Opt. Soc. Am. B 35, 95-99 (2018).

25. A. D. Sánchez, P. I. Fierens, S. M. Hernandez, J. Bonetti, G. Brambilla, and D. F. Grosz, "Anti-stokes raman gain enabled by modulation instability in mid-ir waveguides," J. Opt. Soc. Am. B 35, 2828-2832 (2018).

26. P. Wang, G. Brambilla, M. Ding, X. Zhang, Y. Semenova, Q. Wu, and G. Farrell, "An sms fiber structure based on chalcogenide multimode fiber," in Nonlinear Optics and Applications VI, , vol. 8434 (International Society for Optics and Photonics, 2012), p. 84340N.

27. P. Wang, G. S. Murugan, G. Brambilla, M. Ding, Y. Semenova, Q. Wu, and G. Farrell, "Chalcogenide microsphere fabricated from fiber tapers using contact with a high-temperature ceramic surface," IEEE Photon. Technol. Lett 24, 1103-1105 (2012).

28. A. Hasegawa and W. Brinkman, "Tunable coherent IR and FIR sources utilizing modulational instability," IEEE J. Quantum Electron. 16, 694697 (1980).

29. K. Tai, A. Hasegawa, and A. Tomita, "Observation of modulational instability in optical fibers," Phys. Rev. Lett. 56, 135-138 (1986).

30. A. Demircan and U. Bandelow, "Supercontinuum generation by the modulation instability," Opt. Commun. 244, 181-185 (2005).

31. J. M. Dudley, G. Genty, F. Dias, B. Kibler, and N. Akhmediev, "Modulation instability, Akhmediev breathers and continuous wave supercontinuum generation," Opt. Express 17, 21497-21508 (2009).

32. D. Solli, C. Ropers, P. Koonath, and B. Jalali, "Optical rogue waves," Nature 450, 1054-1057 (2007).

33. K. Hammani, C. Finot, B. Kibler, and G. Millot, "Soliton generation and rogue-wave-like behavior through fourth-order scalar modulation instability," Photonics Journal, IEEE 1, 205-212 (2009).

34. N. Akhmediev, J. M. Soto-Crespo, and A. Ankiewicz, "How to excite a rogue wave," Phys. Rev. A 80, 043818 (2009).

35. S. T. Sørensen, C. Larsen, U. Møller, P. M. Moselund, C. L. Thomsen, and $\mathrm{O}$. Bang, "Influence of pump power and modulation instability gain spectrum on seeded supercontinuum and rogue wave generation," J. Opt. Soc. Am. B 29, 2875-2885 (2012).

36. P. Béjot, B. Kibler, E. Hertz, B. Lavorel, and O. Faucher, "General approach to spatiotemporal modulational instability processes," Phys. Rev. A 83, 013830 (2011).

37. J. Bonetti, S. M. Hernandez, P. I. Fierens, and D. F. Grosz, "Analytical study of coherence in seeded modulation instability," Phys. Rev. A 94 033826 (2016)

38. S. M. Hernandez, P. I. Fierens, J. Bonetti, A. D. Sánchez, and D. F. Grosz, "A geometrical view of scalar modulation instability in optical fibers," IEEE Photonics J. 9, 1-8 (2017).

39. A. Rieznik, A. Heidt, P. Konig, V. Bettachini, and D. Grosz, "Optimum integration procedures for supercontinuum simulation," IEEE Photonics J. 4, 552-560 (2012). 